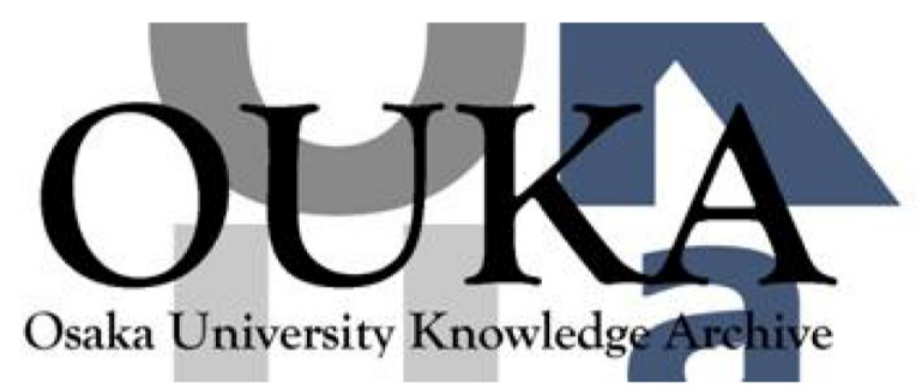

\begin{tabular}{|c|l|}
\hline Title & $\begin{array}{l}\text { Distribution of light-induced defect states in } \\
\text { undoped amorphous silicon }\end{array}$ \\
\hline Author(s) & $\begin{array}{l}\text { Hattori, Kiminori; Anzai, Makoto; Okamoto, } \\
\text { Hiroaki et al. }\end{array}$ \\
\hline Citation & Journal of Applied Physics. 77(7) p. 2989-p. 2992 \\
\hline Issue Date & $1995-04-01$ \\
\hline oaire:version & VoR \\
\hline URL & https://hdl.handle.net/11094/3048 \\
\hline rights & \\
\hline Note & \\
\hline
\end{tabular}

Osaka University Knowledge Archive : OUKA

https://ir. Library. osaka-u. ac. jp/

Osaka University 


\title{
Distribution of light-induced defect states in undoped amorphous silicon
}

\author{
Kiminori Hattori, a) Makoto Anzai, Hiroaki Okamoto, and Yoshihiro Hamakawa \\ Faculty of Engineering Science, Osaka University, Toyonaka, Osaka 560, Japan
}

(Received 30 September 1994; accepted for publication 1 December 1994)

\begin{abstract}
Distribution of light-induced defect states in undoped amorphous silicon has been studied by using the modulated photoconductivity spectroscopy technique. The experimental results show that a large increase of the neutral defect states occurs, and the positively charged defect states grow particularly in the midgap energy range. The qualitative features of the measured energy distribution agree well with the theoretical prediction from the current defect formation model, although a quantitative comparison with respect to the magnitude of density-of-states reveals a discrepancy between theory and experiment. (-) 1995 American Institute of Physics.
\end{abstract}

\section{INTRODUCTION}

The light-induced metastable changes in hydrogenated amorphous silicon $(a-\mathrm{Si}: \mathrm{H})$ have been the subject of intensive study over the last decade. It has been established that intense light illumination produces additional midgap defects, which are ascribed mainly to Si dangling bonds (DBs), and that the light soaked (LS) state returns to thermal equilibrium (TE) state by annealing. However, a detailed understanding of the phenomenon is still missing. In particular, one wishes to know how the defect states change in energy distribution. The change suggested from defect formation model $^{1}$ has not been confirmed experimentally. In this work reported here, the TE and LS defect states in undoped $a-\mathrm{Si}: \mathrm{H}$ have been investigated by applying modulated photoconductivity (MPC) spectroscopy. ${ }^{2}$ The model prediction is examined from a detailed density-of-states comparison between them.

\section{EXPERIMENTAL DETAILS}

The sample studied in this work was a rf-glow-discharge undoped $a-\mathrm{Si}: \mathrm{H}$ film deposited on a glass substrate. The substrate temperature during the deposition was $300^{\circ} \mathrm{C}$ and the film thickness was $1.5 \mu \mathrm{m}$. Coplanar Al electrodes $15 \mathrm{~mm}$ long were evaporated on the top of the film, with the electrode spacing being $1 \mathrm{~mm}$.

The TE state was attained by annealing the sample at $200^{\circ} \mathrm{C}$ for $240 \mathrm{~min}$ in vacuum and cooling it slowly to room temperature. The Fermi-level position of the TE sample was determined to be $\epsilon_{F}=\epsilon_{c}-0.7 \mathrm{eV}$ from the room temperature dark conductivity using the prefactor of $\sigma_{0}=150 \mathrm{~S} \mathrm{~cm}^{-1}$. The LS state was achieved by exposing the sample at roum temperature to a homogeneously absorbed red light from an He-Ne laser $(632.8 \mathrm{~nm})$ with an intensity of $150 \mathrm{~mW} \mathrm{~cm}{ }^{-2}$ for $1020 \mathrm{~min}$. For such a prolonged illumination, it is expected that a light-induced creation of defects is nearly saturated and defect structure comes into the metastable steady state, as suggested from previous experiments. ${ }^{4}$ The light soaking brought about a decrease in photoconductivity, the initial and final values of which, assuming photoconduction dominated by electrons, corresponds to the quasi-Fermi levels at $\epsilon_{f n}=\epsilon_{c}-0.39 \mathrm{eV}$ and $\epsilon_{c}-0.44 \mathrm{eV}$, respectively.

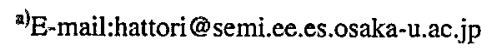

In the MPC measurements, the sample was mounted in a temperature-controlled optical cryostat. The measurement temperature was varied in the range between 140 and $320 \mathrm{~K}$. A light-emitting diode with peak fluxes at $655 \mathrm{~nm}$ modulated at a frequency $\omega=2 \pi f$ with $f$ varying between $0.63 \mathrm{~Hz}$ and $1 \mathrm{kHz}$ was used to generate MPC.

Measurement of the modulus $\sigma_{\omega}$ and phase shift $\phi$ of a low-illumination MPC that reflects electron trapping and detrapping processes gives a density-of-states spectroscopy. Suppose that the density-of-states is made of various types of defects labeled by $i$, each with electron capture coefficient $C_{n}^{i}$, the defect distribution $D^{i}(\epsilon)$ can be related to MPC as ${ }^{2}$

$$
S \equiv-(2 / \pi k T)\left[e \mu_{n} G_{\omega} \sin (\phi) / \sigma_{\omega}+\omega\right]=\Sigma_{i} C_{n}^{i} D^{i}\left(\epsilon_{\omega}^{i}\right),
$$

with $\epsilon_{\omega}^{i}=\epsilon_{c}-k T \ln \left(N_{c} C_{n}^{i} / \omega\right)$. Here, $k T$ is the thermal energy, $e$ the electronic charge, $\mu_{n}\left(=10 \mathrm{~cm}^{2} / \mathrm{V} \mathrm{s}\right)$ the free electron mobility, $\epsilon_{\mathrm{c}}$ the conduction-band (CB) edge, $N_{c}\left(=10^{20}\right.$ $\mathrm{cm}^{-3}$ ) the effective density of $\mathrm{CB}$ states, and $G_{\omega}$ the alternating part of photogeneration rate. The MPC response is conditioned by the electron concentration $n_{0}$ generated by the background steady-state illumination $G_{0}$, since MPCactive are only the defect states lying above $\epsilon_{f n}=\epsilon_{c}-k T \ln \left(N_{c} / n_{0}\right)$ at which thermal emission of a trapped electron preferentially takes place rather than recombination with a hole. Due to this limitation, for a fixed $\omega$ and $T$, the signal $S$ scanned with $n_{0}$ exhibits a falloff when $n_{0} C_{n}^{i}>\omega$. Measurement of the evolution $S\left(n_{0}\right)$ thus allows us to determine $C_{n}^{i}$ as well as $D^{i}(\epsilon)$. For $a-\mathrm{Si}: \mathrm{H}$ material containing DB defects, each of which has three states of charge, $D^{+}, D^{0}$, and $D^{-}$, the distribution of the $+/ 0$ transition levels $D^{+/ 0}(\epsilon)$, associated with the capture coefficient for $D^{+}\left(C_{n}^{+}\right)$, and the distribution of the $0 /-$ transition levels $D^{0 \prime}(\epsilon) \rightarrow D^{0 \prime}-(\epsilon)$, associated with the capture coefficient for $D^{0}\left(C_{n}^{0}\right)$, can be separately obtaincd. Details of the evaluation procedure were described in our previous article. ${ }^{2}$

\section{MODEL PREDICTION}

Before going into the description of the MPC spectroscopy results, it may be appropriate to summarize what kind of light-induced change in $\mathrm{DB}$ defect distribution is expected from the current defect formation model. ${ }^{1,5}$ Assumed in the model is that the DB defects are created by the breaking of weak $\mathrm{Si}-\mathrm{Si}$ bonds, which are relevant to the tail states dis- 
tributed like $\exp \left[-\left(\epsilon-\epsilon_{v}\right) / k T_{v}\right]$ above the valence band edge $\epsilon_{v}$. The defect formation may be stabilized by a diffusive $\mathrm{H}$ motion through breaking and reforming $\mathrm{Si}-\mathrm{H}$ bonds. When a distribution $P(\epsilon)$ of potential energy sites at which defects could be formed, the so-called defect pool, is present due to the inherent disorder of the amorphous network, the resulting defect distribution $D(\epsilon)$ is approximately represented as

$$
D(\epsilon)=\gamma P(\epsilon)\left\{\exp \left[-\left(\epsilon-\epsilon_{v}\right) / k T\right] / f^{0}\right\}^{T / T^{*}} .
$$

In the above expression, $\gamma$ is the energy-independent scaling factor, $f^{0}$ the occupation function for $D^{0}$, and $T^{*}=T_{v}+m T / 2$, where $m$ denotes the number of $\mathrm{Si}-\mathrm{H}$ bonds mediating the defect-forming reaction. The TE defect states with distribution $D_{\mathrm{TE}}(\epsilon)$ and the LS defect states with distribution $D_{\mathrm{LS}}(\epsilon)$ are both originated from the same defect pool represented by $P(\epsilon)$. The difference in spectral shape of $D(\epsilon)$ is provided by the function $\left\{\exp \left[-\left(\epsilon-\epsilon_{v}\right) / k T\right] /\right.$ $\left.f^{0}\right\}^{\text {TiT* }}$.

It is well known that the occupation function $f^{0}$ is given at dark equilibrium by

$$
\begin{aligned}
f^{0}= & \left\{1+\exp \left[\left(\epsilon-\epsilon_{F}\right) / k T\right] / 2\right. \\
& \left.+\exp \left[\left(\epsilon_{F}-\epsilon-U\right) / k T\right] / 2\right\}^{-1},
\end{aligned}
$$

where $U$ is the effective correlation energy that separates $D^{+10}(\epsilon)[=\mathrm{D}(\epsilon)]$ and $D^{0 /-}(\epsilon)[=D(\epsilon+U)]$. A more general expression that can deal with nonequilibrium cases is derived from the detailed balance of electronic transition processes; ${ }^{6}$

$$
\begin{aligned}
f^{0}= & {\left[1+\left(p C_{p}^{0}+e_{n}^{0}\right) /\left(n C_{n}^{+}+e_{p}^{+}\right)\right.} \\
& \left.+\left(n C_{n}^{0} e_{p}^{0} \rightarrow n C_{n}^{0}+e_{p}^{0}\right) /\left(p C_{p}^{-}+e_{n}^{-}\right)\right]^{-1},
\end{aligned}
$$

where $e_{n}^{0,-}$ are the electron thermal emission rates for $D^{0,-}$ states, $e_{p}^{+, 0}$ the hole thermal emission rates for $D^{+, 0}$ states, and $C_{n}^{+, 0}$ and $C_{p}^{0,-}$ the associated capture coefficients. Applying a regional approximation in energy to Eqs. (2) and (3), the relative change in defect distribution $D_{\mathrm{LS}}(\epsilon) / D_{\mathrm{TE}}(\epsilon)$ is found to be expressed as

$$
\begin{aligned}
& R \exp \left[-\left(\epsilon_{f n}-\epsilon_{v}\right) /\left.k T^{*}\right|_{\mathrm{LS}}+\left(\epsilon_{F}-\epsilon_{v}\right) /\left.k T^{*}\right|_{\mathrm{TE}}\right] \\
& \quad \text { for } \epsilon>\left.\epsilon_{t n}^{0}\right|_{\mathrm{LS}}, \\
& R \exp \left[-\left(\epsilon-\epsilon_{v}\right) /\left.k T^{* *}\right|_{\mathrm{LS}}+\left(\epsilon_{F}-\epsilon_{v}\right) /\left.k T^{*}\right|_{\mathrm{TE}}\right] \\
& \quad \text { for }\left.\epsilon_{t n}^{0}\right|_{\mathrm{LS}}>\epsilon>\left.\epsilon_{F}\right|_{\mathrm{TE}}, \\
& R \exp \left[-\left(\epsilon-\epsilon_{v}\right) /\left.k T^{*}\right|_{\mathrm{LS}}+\left(\epsilon-\epsilon_{v}\right) /\left.k T^{*}\right|_{\mathrm{TE}}\right] \\
& \quad \text { for }\left.\epsilon_{F}\right|_{\mathrm{TE}}>\epsilon>\left.\epsilon_{F}\right|_{\mathrm{TE}}-U, \\
& R \exp \left[-\left(\epsilon-\epsilon_{v}\right) /\left.k T^{*}\right|_{\mathrm{LS}}-\left(\epsilon_{F}-2 \epsilon-U\right) /\left.k T^{*}\right|_{\mathrm{TE}}\right] \\
& \quad \text { for }\left.\epsilon_{F}\right|_{\mathrm{TE}}-U>\epsilon>\left.\epsilon_{t p}^{0}\right|_{\mathrm{LS}}, \\
& R \exp \left[\left(\epsilon_{f p}-2 \epsilon-U\right) /\left.k T^{*}\right|_{\mathrm{LS}}-\left(\epsilon_{F}-2 \epsilon-U\right) /\left.k T^{*}\right|_{\mathrm{TE}}\right] \\
& \quad \text { for }\left.\epsilon_{t p}^{0}\right|_{\mathrm{LS}}>\epsilon,
\end{aligned}
$$

where $R$ is defined as $\left(\left.y\right|_{\mathrm{LS}} /\left(\left.\gamma\right|_{\mathrm{TE}}\right) \rightarrow\left(\left.\gamma\right|_{\mathrm{LS}} /\left(\left.\gamma\right|_{\mathrm{TE}}\right)\right.\right.$ and the notations $\left.\right|_{\mathrm{TE}, \mathrm{LS}}$ designate the quantities under the TE and LS defect-forming conditions, respectively. Although the above expressions are obtained with assuming that $\left.(1 / 2)^{T / T^{*}}\right|_{\mathrm{TE}, \mathrm{LS}}$

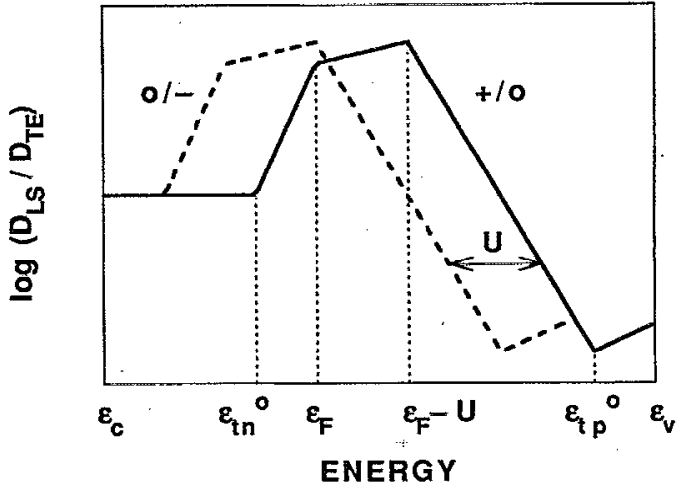

FIG. 1. Schematic illustration of function $D_{\mathrm{LS}}(\epsilon) / D_{\mathrm{TE}}(\epsilon)$. The energy $\epsilon_{F}$ is the dark Fermi level under the TE defect-forming condition. The energies $\epsilon_{t n, p}^{0}$ are the quasi-Fermi levels for carriers trapped at $D^{0}$ states under the LS defect-forming condition.

and $\left.\left(1+p C_{p}^{0} / n C_{n}^{+}+n C_{n}^{0} / p C_{p}^{-}\right)^{T / T^{* *}}\right|_{\text {LS }}$ are on the order of unity, the simplification does not significantly alter the discussion described below. The energies $\epsilon_{t n}^{0}=\epsilon_{c}-k T \ln \left[N_{c} C_{n}^{+} / 2\left(n C_{n}^{+}+p C_{p}^{0}\right)\right] \quad$ and $\quad \epsilon_{t p}^{0}=\epsilon_{v}-U$ $+k T \ln \left[N_{v} C_{p}^{-} / 2\left(n C_{n}^{0}+p C_{p}^{-}\right)\right]$are the quasi-Fermi levels for electrons and holes trapped at $D^{0}$ states introduced by Longeaud and Kleider. ${ }^{6}$ The coefficient $R$ determines the change in defect density. The suggested theoretical formulation ${ }^{1}$ involves concentration of minority photocarriers $\left.p\right|_{\text {LS }}$, which is hardly assessed from experiments. Apart from the absolute scale of $D_{\mathrm{LS}}(\epsilon) / D_{\mathrm{TE}}(\epsilon)$, we shall restrict our attention to its energy profile in the following.

Figure 1 displays the expected spectral shape for $D_{\mathrm{LS}}(\epsilon) / D_{\mathrm{TE}}(\epsilon)$. As found from the illustration, a lightsoaking yields a defect distribution with a largest growth in the midgap range $\left.\epsilon_{F}\right|_{\mathrm{TE}}>\epsilon>\left.\epsilon_{F}\right|_{\mathrm{TE}}-U$ where $D^{0}$ occurs in the TE state. The MPC spectroscopy measures electrontrapping states lying in the CB side, indicating that a close look at this energy region is of special importance for a comparison between the theory and the experiment. For the $+/ 0$ transition levels, the ratio $D_{\mathrm{LS}}(\epsilon) / D_{\mathrm{TE}}(\epsilon)$ are kept constant above $\left.\epsilon_{t n}^{0}\right|_{L S}$, while below $\epsilon_{t n}^{0} h_{S S}$ it shows an exponential increase. Between $\left.\epsilon_{F}\right|_{\mathrm{TE}}$ and $\left.\epsilon_{F}\right|_{\mathrm{TE}}-U$, the ratio varies exponentially but its slope is relatively small. The behavior for the $0 /-$ transition levels follows that for the $+/ 0$ transition levels, being shifted by $U$.

\section{RESULTS AND DISCUSSION}

We will here proceed to discussion on experimental results. Several repeated MPC measurements for both TE and LS samples have been carried out, yielding estimates for the capture coefficients in the range $C_{n}^{+}=(0.8-1.4) \times 10^{-6}$ $\mathrm{cm}^{3} \cdot \mathrm{s}^{-1}$ and $C_{n}^{0}=(0.3-1.4) \times 10^{-8} \mathrm{~cm}^{3} \mathrm{~s}^{-1}$. A general trend that a charged defect is more efficient in capturing a carrier than the neutral one due to Coulomb attraction underlies the identification of charge states of defects. The deviation among estimated values, which is thought to be partly due to some ambiguity in deconvoluting the measured MPC signal into the +10 and $0 /-$ transition components, affects little the energy profile of derived defect distribution. 


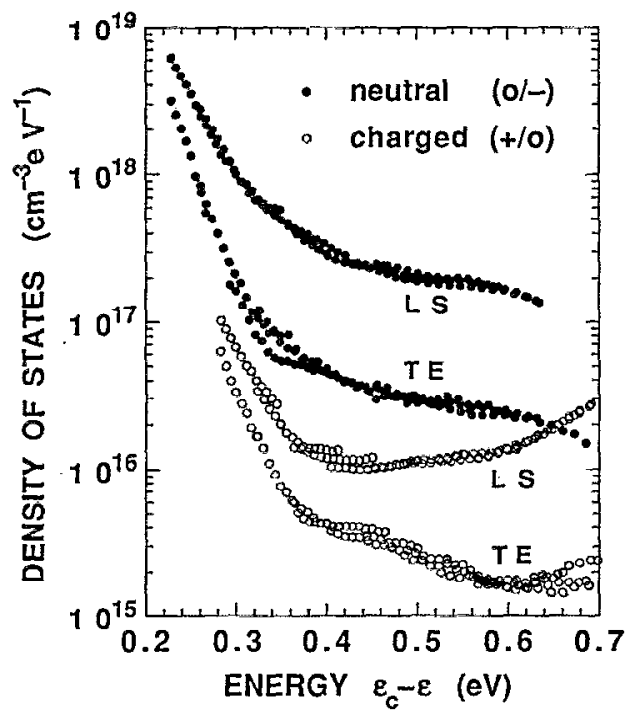

FIG. 2. LS and TE defect distributions measured by MPC spectroscopy.

The results plotted in Fig. 2 were obtained with $C_{n}^{+}=10^{-6} \mathrm{~cm}^{3} \mathrm{~s}^{-1}$ and $C_{n}^{(0}=10^{-8} \mathrm{~cm}^{3} \mathrm{~s}^{-1}$, which differ slightly from those reported in our previous publication. ${ }^{2}$ Deep defect bands located around $0.4-0.6 \mathrm{eV}$ below $\mathrm{CB}$ are assigned to arise from the positively charged and neutral DB states and represent distributions $D^{+/ 0}(\epsilon)$ and $D^{0 /-}(\epsilon)$, respectively. The capture coefficients, being almost identical with $C_{n}^{+, 0}$, were assessed for the exponential distributions superimposed on $D B$ defect bands, which are likely to be associated with $C B$ tail states. The positively charged state of the CB tail is highly improbable, however, a specific subset of tail states that are built in close to $D^{+}$and exposed to Coulomb attractive potential produced by the charged center might possess capture coefficient as large as $C_{n}^{+}{ }^{2}$

In order to compare the MPC results with the defect formation model, we plot the ratio of density of LS defect states to density of TE defect states in Fig. 3. The relative change in distribution falls down and approaches unity for $\epsilon>\epsilon_{r}-0.3 \mathrm{eV}$, where the $\mathrm{DB}$ defect distribution is masked behind the $\mathrm{CB}$ tail distribution. The spectrum below $\epsilon_{c}-0.4$ $\mathrm{eV}$ is almost flat for the $0 /-$ transition levels, while for the $+/ 0$ transition levels it exponentially grows below a kink at $\epsilon_{r}-0.44 \mathrm{eV}$. The kink position coincides with the quasiFermi level $\left.\epsilon_{f n}\right|_{\text {LS }}$. Note that $\epsilon_{t n}^{0} \cong \epsilon_{f n}$ when $n C_{n}^{+} \gg p C_{p}^{0}$. The inequality of capture rate is consistent with a generally accepted view, that is, electrons are majority photocarriers in undoped $a$-Si:H, and a charged defect center exceeds the neutral one in capture efficiency. The observed spectral shapes are thus in reasonable agreement with the theoretical prediction. A small bump at $\epsilon_{\mathrm{r}}-0.34 \mathrm{eV}$ for the $0 /-$ transition levels would imply that a distribution of defect states of different origin is overlapped. A suggestion from previous MPC works ${ }^{2,7}$ is that they are not bulk defects but some surface or interface defects.

For a further quantitative examination of the relative change in distribution, it is convenient to use the ratio for the

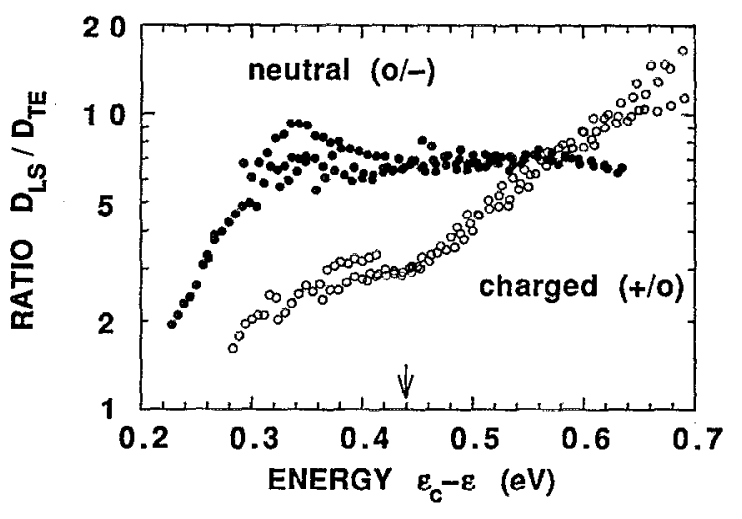

FIG. 3. Ratio of density of LS defect states to density of TE defect states obtained from the results presented in Fig. 2. The arrow indicates the energy position of quasi-Fermi level $\left.\epsilon_{f n}\right|_{L S}$.

$+/ 0$ transition levels at $\left.\epsilon_{t n}^{0}\right|_{L S}$ as a reference. Assuming that $U \cong 0.3 \mathrm{eV},\left.\epsilon_{f n}\right|_{\mathrm{LS}}-\left.\epsilon_{\mathrm{F}}\right|_{\mathrm{TE}} \cong 0.3 \mathrm{eV},\left.k T^{*}\right|_{\mathrm{TE}}-60-100 \mathrm{meV}, 2,5$ and $\left.k T^{*}\right|_{L S}=60-70 \mathrm{meV}$, expected from the model is a prominent growth of $D^{0}$. The resulting ratio for the $+/ 0$ transition levels around $\left.\epsilon_{F}\right|_{T E}$ is $100-1000$ times as large as the reference. However, this was not observed. Experimentally, the increment factor is less than 10, as found from Fig. 3.

The defect formation model with a Gaussian pool $P(\epsilon) \propto \exp \left[-\left(\epsilon-\epsilon_{p}\right)^{2} / 2 \sigma_{p}^{2}\right]$ leads to comparable densities for the charged and neutral DBs in the TE state of undoped material when applying estimates of $\sigma_{p}=0.15 \mathrm{eV}$ and $U=0.3 \mathrm{eV} .{ }^{2}$ By contrast, our observation is that TE- $D^{0}$ density is roughly one order of magnitude larger than TE- $D^{+}$ density. The disagreement must be correlated with the small increment factor in $D_{\mathrm{LS}}(\epsilon) / D_{\mathrm{TE}}(\epsilon)$ described above. The current model leads us to expect that a light initial $n$-type doping and a resulting Fermi level shift, which could arise from contamination of the film with $\mathrm{O}$ or $\mathrm{N}$ atoms, yields a larger ratio of TE- $D^{0}$ to TE- $D^{+}$density. ${ }^{5}$ It should be noted, however, that the possible $\left.\epsilon_{F}\right|_{T E}$ shift does not essentially alter the formulation for ratio $D_{\mathrm{LS}}(\epsilon) / D_{\mathrm{TE}}(\epsilon)$ described in Sec. III, and does not give an explanation for our observation. Interpretation for present experimental results might need some corrections for theoretical description of the $\mathrm{TE}$ process, which are not clear at present and are the subject of further investigations.

\section{SUMMARY}

MPC spectroscopy has been applied to investigate the distribution of light-induced defect states in undoped $a-\mathrm{Si}: \mathrm{H}$. It is shown from the measurements that a large increase of the neutral defect states occurs, and the positively charged defect states grow particularly in the midgap energy range. The qualitative features of the measured energy distribution agree well with the theoretical prediction from the current defect formation model, although a quantitative comparison with respect to the magnitude of density-of-states reveals a discrepancy between theory and experiment. 
The results reported here were all obtained with a single sample. The fact that the correlation between TE and LS defect distributions observed was found for a randomly chosen high-quality sample, which has TE- $D^{0}$ density of about $10^{16} \mathrm{~cm}^{-3}$ (see Fig. 2), suggests that other samples of the same type would give similar results. Our more general experience with other samples does not lead us to expect significant variations in the defect distribution between samples. It is hoped that this work will provide incentive for researchers to make density-of-states measurements on TE and LS undoped $a-\mathrm{Si}: \mathrm{H}$ samples, to test the generality of present results, as well as to refine the theoretical description for defect formation.

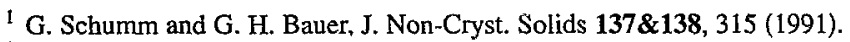

${ }^{2}$ K. Hattori, Y. Adachi, M. Anzai, H. Okamoto, and Y. Hamakawa, J. Appl. Phys. 76, 2841 (1994).

${ }^{3}$ J. Stuke, J. Non-Cryst. Solids 97\&98, 1 (1987).

${ }^{4}$ M. S. Brandt and M. Stutzmann, J. Appl. Phys. 75, 2507 (1994).

${ }^{5}$ M. J. Powell and S. C. Deane, Phys. Rev. B 48, 10815 (1993).

${ }^{6}$ C. Longeaud and J. P. Kleider, Phys. Rev. B 48, 8715 (1993).

${ }^{7}$ J. P. Kleider, C. Longeaud, and P. Roca i Cabarrocas, J. Appl. Phys. 72, 4727 (1992). 